\title{
BMJ Open Dexmedetomidine for the prevention of postoperative delirium in patients after intracranial operation for brain tumours (DEPOD study): a study protocol and statistical plan for a multicentre randomised controlled trial
}

Xuan He, ${ }^{1}$ Kun-Ming Cheng, ${ }^{1}$ Linlin Zhang, ${ }^{1}$ Hongqiu Gu $\left(\mathbb{D},{ }^{2}\right.$ Xin Qu, ${ }^{3}$ Yuan Xu, ${ }^{4}$ Penglin Ma (D) , ${ }^{5}$ Jian-Xin Zhou (D) ${ }^{1}$

To cite: He X, Cheng K$M$, Zhang L, et al. Dexmedetomidine for the prevention of postoperative delirium in patients after intracranial operation for brain tumours (DEPOD study): a study protocol and statistical plan for a multicentre randomised controlled trial. BMJ Open 2020;10:e040939. doi:10.1136/ bmjopen-2020-040939

- Prepublication history for this paper is available online. To view these files, please visit the journal online (http://dx.doi org/10.1136/bmjopen-2020040939).

Received 26 May 2020 Revised 13 0ctober 2020 Accepted 08 November 2020

Check for updates

(c) Author(s) (or their employer(s)) 2020. Re-use permitted under CC BY-NC. No commercial re-use. See rights and permissions. Published by BMJ.

For numbered affiliations see end of article.

Correspondence to Professor Jian-Xin Zhou; zhoujx.cn@icloud.com

\section{ABSTRACT}

Introduction Postoperative delirium (POD) is prevalent in patients after major surgery and is associated with adverse outcomes. Several studies have reported that dexmedetomidine, a highly selective $\alpha 2$-adrenergic receptor agonist, can decrease the incidence of POD. However, neurosurgical patients are usually excluded from previous studies. The present study was designed to investigate the impact of prophylactic use of low-dose dexmedetomidine on the incidence of POD in patients after intracranial operation.

Methods and analysis This is a multicentre, randomised, double-blinded and placebo-controlled trial. Seven hundred intensive care unit admitted patients after elective intracranial operation for brain tumours under general anaesthesia are randomly assigned to the dexmedetomidine group or the placebo group with a $1: 1$ ratio. For patients in the dexmedetomidine group, a continuous infusion of dexmedetomidine will be started at a rate of $0.1 \mu \mathrm{g} / \mathrm{kg} / \mathrm{hour}$ immediately after enrolment on the day of operation and continued until 08:00 on postoperative day 1. For patients in the placebo group, normal saline will be administered at the same rate as in the dexmedetomidine group. The patients will be followed up for 28 days after enrolment. The primary endpoint is the incidence of POD, which is assessed two times per day using the Confusion Assessment Method for the intensive care unit (ICU), during the first 5 postoperative days. The secondary endpoints include the incidence of dexmedetomidine-related adverse events and nondelirium complications, the length of stay in the ICU and hospital and all-cause 28-day mortality after the operation. Ethics and dissemination The study protocol was approved by the Institutional Review Board of Beijing Tiantan Hospital Affiliated to Capital Medical University (No KY2019-091-02) and registered at ClinicalTrials.gov. The results of the trial will be presented at national and international conferences relevant to subject fields and submitted to international peer-reviewed journals. Trial registration number Trial registration number: NCT04399343; Pre-results.

\section{Strengths and limitations of the study}

- The study design will be multicentre, randomised, double-blinded and placebo-controlled, with a relatively large sample size.

- To the best of our knowledge, this is the first study to evaluate the effect of prophylactic use of dexmedetomidine on postoperative delirium (POD) in neurosurgical patients.

- Only intensive care unit admitted patients are included, which will limit the generalisability of the results.

- Only early outcomes are assessed including the incidence of POD and other endpoints up to 28 days after the operation.

- Although patients with medical records documented preoperative history of mental or cognitive disorders are excluded, no systematic cognitive assessment will be performed before the intracranial operation.

\section{BACKGROUND}

Postoperative delirium (POD) is one of the main postoperative complications after major surgery, especially in patients admitted to the intensive care unit (ICU). ${ }^{12}$ Studies have shown that POD is associated with adverse outcomes, including prolonged length of stay in the ICU and hospital, higher morbidity and mortality risks, increased healthcare costs, and reduced long-term quality of life..$^{3-5}$ However, patients with neurological illness are usually excluded from previous researches, and delirium is underestimated due to potential consciousness and cognition impairment. ${ }^{67}$ Recently, more attention has been devoted to POD in patients after neurosurgery. ${ }^{8-11}$ In our cohort with 800 adult patients admitted to the ICU after elective intracranial 
operation under general anaesthesia, delirium was screened within 3 days after the surgery. ${ }^{8}$ The incidence of POD is about $20 \%$, which is comparable to the results in the control group of randomised controlled trials with non-neurosurgical patients $(18 \%-21 \%) \cdot{ }^{12-14}$ Potential associations between POD and adverse outcomes have also been reported in neurosurgical patients. ${ }^{8-11}$ These results indicate that early prevention of POD should be employed in this population.

Perioperative preventive medications have been an active area of clinical POD researches. ${ }^{15}$ As a highly selective $\alpha_{2}$-adrenergic receptor agonist, dexmedetomidine has been investigated as a preventive agent for POD. ${ }^{12-14}$ Systematic reviews and meta-analyses of randomised controlled trials have shown that perioperative administration of dexmedetomidine may decrease the incidence of POD,${ }^{12}$ in patients after either cardiac ${ }^{13}$ or non-cardiac surgery. ${ }^{14}$ However, neurosurgical patients are often excluded in randomised controlled trials of POD. ${ }^{12} 14$

In a randomised controlled trial in patients with delayed extubation after craniotomy, ${ }^{16}$ we found that early prophylactic use of dexmedetomidine during the first 24 hours after the operation significantly reduced the incidence of agitation, which might include some hyperactive delirium. In this study, an infusion rate of dexmedetomidine was set at $0.6 \mu \mathrm{g} / \mathrm{kg} /$ hour, which did not produce respiratory depression but increased the incidence of bradycardia. In a recent randomised controlled trial in elderly patients after non-cardiac surgery and admitted to the ICU, prophylactic use of low-dose $(0.1 \mu \mathrm{g} / \mathrm{kg} / \mathrm{hour})$ dexmedetomidine significantly decreased the incidence of POD without the increased occurrence of hypotension and bradycardia. ${ }^{17} \mathrm{~A}$ randomised controlled trial in critically ill adults also demonstrated that nocturnal administration of low-dose dexmedetomidine reduced the incidence of delirium during the ICU stay. ${ }^{18}$

The above findings encouraged us to design a multicentre randomised controlled trial, DExmedetomidine for the prevention of PostOperative Delirium, aiming to evaluate the efficacy and safety of low-dose dexmedetomidine for prevention of POD in patients after intracranial operation for brain tumours. The primary hypothesis is that, compared with the placebo group, the prophylactic use of low-dose dexmedetomidine can decrease the incidence of POD without significant adverse events in this population.

\section{METHODS AND ANALYSIS \\ Study design}

This prospective, multicentre, randomised, doubleblinded and placebo-controlled trial with two parallel arms was designed to test whether the prophylactic use of low-dose dexmedetomidine would decrease the incidence of POD in adult patients admitted to the ICU after elective intracranial operation for brain tumours. The design of the present study has adhered to the standard protocol items for randomised trials. ${ }^{19}$

The flow chart of the trial is shown in figure 1.

\section{Patient and public involvement}

Patients and the public are not involved in the design or conduct of the study. There is no plan to disseminate our results to study participants.

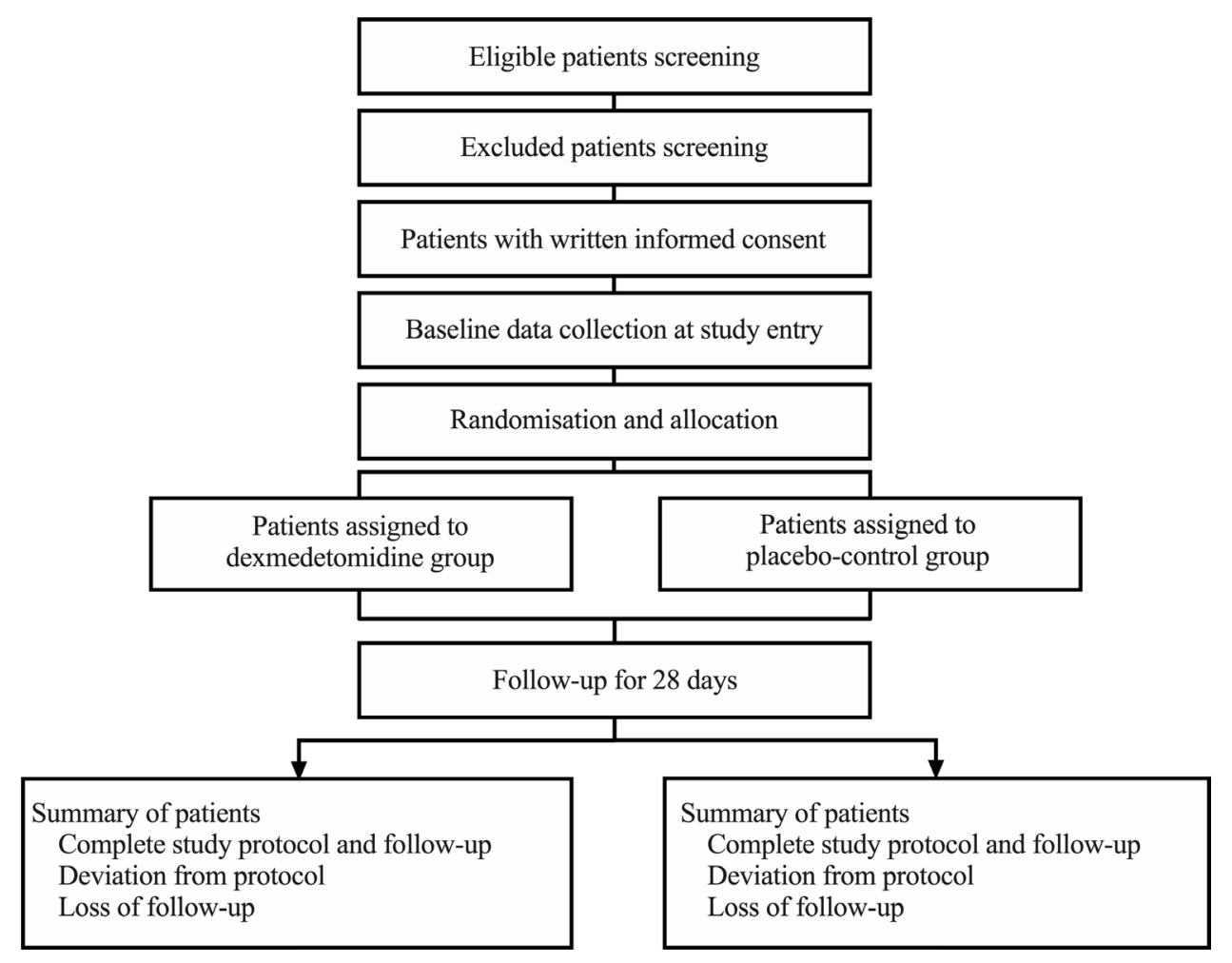

Figure 1 Flow chart of the trial. 


\section{Study locations}

The study will be conducted in five ICUs in four teaching hospitals including Beijing Tiantan hospital (one general ICU and one neurosurgical ICU with a total of 50 beds) and Xuanwu hospital (one neurosurgical ICU with 30 beds) affiliated to Capital Medical University, Peking University Third Hospital (one general ICU with 30 beds) and Beijing Tsinghua Changgung Hospital affiliated to Tsinghua University (one general ICU with 20 beds), in Beijing, China.

\section{Participants}

Potential eligible participants are screened on admission to the ICU.

The inclusion criteria are adult patients after elective intracranial operation for brain tumours under general anaesthesia and who are admitted to the ICU directly from the operating room or postoperative care unit.

The exclusion criteria include:

1. Age younger than 18 years.

2. Admitted to the ICU after 22:00.

3. Medical records documented preoperative history of mental or cognitive disorders including schizophrenia, epilepsy, Parkinsonism, or dementia.

4. Medical records documented inability to communicate in the preoperative period due to coma or language barrier.

5. History of drug abuse of psychoactive and anaesthetic drugs.

6. Known preoperative severe bradycardia (lower than 50 beats/min), sick sinus syndrome, second-degree or third-degree atrioventricular block, or left ventricular ejection fraction lower than $30 \%$.

7. Serious hepatic dysfunction (Child-Pugh class C).

8. Severe renal dysfunction requiring renal replacement therapy before the surgery.

9. Allergies to ingredients or components of 5-[(1S)-1(2,3-dimethylphenyl)ethyl]-1H-imidazole (dexmedetomidine hydrochloride).

10. American Society of Anesthesiologists classification of IV-VI.

11. Moribund condition with a low likelihood of survival for more than 24 hours.

12. Pregnancy or lactation women.

13. Current enrolment in another clinical trial.

14. Refusal to participate.

Once the patient's eligibility is confirmed, a 24-hour on-call study coordinator will be contacted. Each relevant aspect of the project will be described and written informed consent will be obtained from the patient. If the patient cannot provide consent, written informed consent will be obtained from their authorised representatives. The study coordinator will be particularly careful to assure the patients or their authorised representatives that they are free to decline consent without consequences, and that they can withdraw consent at any time without an impact on treatment.
Randomisation, allocation concealment and blinding

After obtaining informed consent, the enrolled patients are randomly allocated to receive a continuous intravenous infusion of dexmedetomidine or placebo (normal saline).

To ensure allocation concealment of the randomisation, a password-protected central randomisation system is established and applied. A biostatistician who will not participate in data management and statistical analysis generates random numbers in a 1:1 ratio using the SAS V.9.2 software (SAS Institute). The stratification is performed among the five participating ICUs with prespecified block sizes depending on the anticipated number of recruitments. The random allocation sequence remains concealed from investigators in charge of enrolment. When the patient's enrolment is confirmed, the investigator will $\log$ in to the central randomisation system, complete the checklist of inclusion and exclusion criteria, and acquire the random number. A pharmacist in each centre who has no knowledge of patients before the randomisation and will not participate in the rest of the study provides the study agents according to the randomisation results.

The experimental agents (dexmedetomidine hydrochloride $200 \mu \mathrm{g} / 2 \mathrm{~mL}$ or normal saline $2 \mathrm{~mL}$ ) are manufactured and provided by the Jiangsu Nhwa Pharmaceutical Co., (Jiangsu, China). The agents are packed as clear aqueous solutions with the same character in the same $3 \mathrm{~mL}$ ampoules.

Information on randomisation and group allocation will be blinded from the patients, the investigators who perform data collection and follow-up, the ICU physicians and other healthcare providers who are responsible for patients care. Blinding will be maintained throughout the entire research period.

During the study, group allocation can be unmasked in order to protect the patient's safety. Investigators can request an urgent unmasking to the central randomisation system if considered necessary in case of the occurrence of severe adverse events or any unexpected deterioration of the patient's condition. Because each ampoule containing an experimental drug or placebo has a unique randomisation number, this emergency unmasking will not reveal the group allocations in other enrolled patients. The unmasked patients will be included in the intention-to-treat analysis.

\section{Data collected at study entry}

At baseline, demographic data, history of past illnesses and diagnosis of the patients are obtained. The surgical site and approach, body position, malignant tumour, intraoperative medications, fluid balance during operation, the amount of bleeding and blood transfusion during operation, episode of hypoxemia and hypotension during operation and duration of operation are recorded. The presence of an endotracheal tube and the need for mechanical ventilation on ICU admission are documented. The Acute Physiology and Chronic Health 
Evaluation II Score and Glasgow Coma Scale are calculated. The time interval between the end of operation and study drug infusion is recorded.

Before the start of study agent infusion, delirium is evaluated using the Confusion Assessment Method for the ICU (CAM-ICU) $)^{20-22}$ and documented as emergence delirium.

\section{Trial interventions and management of pain, agitation and delirium}

All enrolled patients will be randomly allocated to receive a continuous intravenous infusion of dexmedetomidine hydrochloride (dexmedetomidine group) or normal saline (placebo group).

The study agent (dexmedetomidine hydrochloride 200 $\mu \mathrm{g} / 2 \mathrm{~mL}$ or normal saline $2 \mathrm{~mL}$ ) is diluted with normal saline to $50 \mathrm{~mL}$ and continuously infused at a rate of $0.025 \mathrm{~mL} / \mathrm{kg} /$ hour. This represents an infusion rate of $0.1 \mu \mathrm{g} / \mathrm{kg} /$ hour dexmedetomidine hydrochloride in the dexmedetomidine group.

The intravenous infusion will be started immediately after enrolment and continued until 08:00 on postoperative day 1 . During the study, scopolamine and penehyclidine will not be given. Open-labelled dexmedetomidine will be prohibited. Atropine can only be applied to treat bradycardia.

Routine clinical managements of pain, agitation and delirium have been established according to the recommendations in guidelines proposed by the American Society of Critical Care Medicine ${ }^{23}$ and the European Society of Anaesthesiology ${ }^{1}$ in all participating ICUs. ${ }^{81624-26}$ During the study protocol group discussion and training, several key elements in comprehensive approaches to reduce POD have been highlighted and emphasised as following:

1. Regular pain assessment is performed using patient's self-reported scales such as the Numeric Rating Scale (NRS) and the Visual Analogue Scale, and if impossible, using behavioural pain scales such as the Behavioral Pain Scale and the Critical-Care Pain Observation Tool (CPOT).

2. Opioids or non-steroidal anti-inflammatory drugs should be used in patients who require analgesia.

3. Regular agitation-sedation assessment is performed using the Richmond Agitation-Sedation Scale (RASS) or the Sedation-Agitation Scale (SAS).

4. If the patients exhibit agitation, sedation should be initiated using common sedatives including propofol and midazolam. Although midazolam is available, it is not recommended as the priority of sedative drugs.

5. A light sedation depth is recommended to maintain an RASS Score of -2 to +1 or an SAS score of 3-5.

6 . In conjunction with the recent clinical guidelines for ICU delirium, ${ }^{27}$ we recommend that repeated reorientation, cognitive stimulation, early mobilisation, sleeppromotion strategies and hearing or vision aids should be performed as long as possible.
7. Patients who develop POD are first given nonpharmacological treatments listed above. Pharmacological treatment is only reserved for patients with hyperactive delirium with disorientated agitation. Haloperidol $(0.5-2 \mathrm{mg})$ can be intravenously injected followed by the administration of half of the loading dose for every 4-6hours until the control of the severe hyperactive delirium.

The routine patient management and ICU discharge will be decided by the responsible ICU physicians. The hospital discharge will be decided by the responsible neurosurgeons.

\section{Adverse events management}

Adverse events will be monitored from the start of study agent infusion until 24 hours or until ICU discharge. Investigators should record all the adverse events including the type, diagnosis time, duration and consequences. Predicted adverse events in the present study include bradycardia (defined as heart rate lower than 50 beats/min), hypotension (defined as systolic blood pressure lower than $90 \mathrm{~mm} \mathrm{Hg}$ ) and hypoxemia (defined as pulse oxygen saturation lower than 90\%). ${ }^{16} 17$

Attending ICU physicians can stop the study agent infusion in the following cases:

1. Heart rate lower than 40 beats/min after atropine in $0.25 \mathrm{mg}$ intravenous bolus.

2. Systolic blood pressure lower than $90 \mathrm{~mm} \mathrm{Hg}$ after fluid resuscitation with $250 \mathrm{~mL}$ crystalloid solution infusion within $15 \mathrm{~min}$.

3. Peripheral pulse oxygen saturation lower than $90 \%$ after administration of oxygen and adjustment of mechanical ventilation for patients without and with endotracheal intubation, respectively.

4. Other conditions deemed necessary by the ICU physician.

In these cases, the reasons that lead to any protocol interruption are documented and reported to the Institutional Review Board of Beijing Tiantan Hospital Affiliated to Capital Medical University within 24 hours. Patients with study protocol interruption will be included in the intention-to-treat analysis.

\section{Outcome measurements}

The patients will be followed up two times per day during the first 5 postoperative days, and then weekly until hospital discharge or until 28 days after the operation. Investigators who are responsible for follow-up will not involve in the study agent infusion and patient care, and they are not allowed to exchange patient's information with ICU physicians taking care of the patients.

The primary endpoint is the incidence of POD during the first 5 postoperative days. ${ }^{1}$ Delirium is evaluated two times per day (08:00 to 10:00 and 18:00 to 20:00) until the fifth postoperative day by the CAM-ICU. ${ }^{20-22}$ Before the initiation of the trial, investigators in each participating ICU are trained to follow the study protocol and to perform the CAM-ICU evaluation. Thereafter, the training will be 
performed at an interval of 3 months during the study. An expert from the department of psychiatry is invited to provide the training. The training courses consist of three phases: (1) group training with information about delirium, RASS and CAM-ICU, as well as a video demonstrating the detailed conduction of CAM-ICU assessment, (2) one-to-one instruction at the bedside and (3) examination on five patients who have been preassessed by the expert. Different results of delirium assessment between the trainee and the expert will be discussed until $100 \%$ agreement is reached.

Secondary endpoints include:

1. Incidence of adverse events from the start of study agent infusion until 24 hours or until ICU discharge.

2. Incidence of non-delirium complications after the operation until 28 days, including airway obstruction and apnoea, respiratory failure, cardiac events, coma, epilepsy, cerebral haemorrhage or infarction, renal injury and infection.

3. Length of stay in the ICU and hospital after the operation.

4. All-cause 28-day mortality after the operation.

Additional prespecified endpoints include the use of sedatives and analgesics during the study agent infusion, pain intensity (assessed by CPOT) ${ }^{23} 27$ and subjective sleep quality (assessed by NRS) ${ }^{28}$ in the morning of postoperative day 1.

\section{Data management and monitoring}

Table 1 shows data collection at each time point.

Before the initiation of the study, an electronic case report form (eCRF) is established and available online at a dedicated website with password-protected access for each participating centre. Each enrolled patient will be assigned an identification number. Patient data will be coded and kept confidential. Related paper records will be stored in a locked cabinet in an access-controlled room.

Each participating centre will indicate a local coordinator in charge of the study. The local coordinators guarantee the integrity of data collection and timely completion of the eCRF.

Data quality and safety will be monitored by an independent Data and Safety Monitoring Board, which will be composed of five multidisciplinary experts who are not have subordinate relationships with any members of the research team. Data management and statistical analysis will be performed by the China National Clinical Research Center for Neurological Diseases in Beijing Tiantan Hospital. Because dexmedetomidine has been widely used in the ICU and its safety has been confirmed in the previous studies, ${ }^{16}{ }^{17}$ an interim analysis will not be scheduled. Severe adverse events will be reported to the Institutional Review Board of Beijing Tiantan Hospital Affiliated to Capital Medical University within 24 hours.

\section{Current sample size justification}

In four observational studies, including 2769 patients after intracranial operation, POD occurred in 349 patients with an overall pooled crude incidence of $13 \% .{ }^{8-11} \mathrm{~A}$ recent meta-analysis of non-cardiac patients showed that the incidence of POD reduced by approximately half when dexmedetomidine was used perioperatively. ${ }^{14}$ We assume that the incidence of POD would be reduced by one-third in the dexmedetomidine group compared with the placebo group in the present study. With a significance and power set at 0.05 (two sided) and $80 \%$, respectively, the sample size required to detect difference is 1088 patients. Taking into account about $5 \%$ of the loss to follow-up rate, 1140 (570 in each group) patients need to be enrolled.

Table 1 Data collection at each time point

\begin{tabular}{|c|c|c|c|}
\hline & At study entry & $\begin{array}{l}\text { Postoperative days } 1-5 \text {, two } \\
\text { times per day }\end{array}$ & $\begin{array}{l}\text { Postoperative days } 7,14,21 \\
\text { and } 28 \text {, once daily }\end{array}$ \\
\hline Demographic data & $\sqrt{ }$ & & \\
\hline Intraoperative data & $\sqrt{ }$ & & \\
\hline Data on ICU admission & $\sqrt{ }$ & & \\
\hline Delirium assessment & $\sqrt{ }$ & $\sqrt{ }$ & \\
\hline Study drug interruption & & $\sqrt{ }$ & \\
\hline Adverse events & & $\sqrt{ }$, if not discharged from ICU & \\
\hline Non-delirium complications & & $\sqrt{ }$ & $\sqrt{ }$, if not discharged from hospital \\
\hline Length of stay in ICU & & $\sqrt{ }$, if not discharged from ICU & $\sqrt{ }$, if not discharged from ICU \\
\hline
\end{tabular}

ICU, intensive care unit. 


\section{Statistical analysis}

All analyses will be conducted according to the intentionto-treat principle, that is, all randomised patients will be analysed in the groups to which they have been originally allocated and will be blinded to treatment assignment.

Categorical variables will be presented as numbers and percentages, and analysed by the $\chi^{2}$ test. Continuous variables will be checked for normal distribution and presented as mean and SD or median and IQR as appropriate. Comparison of continuous variables will be performed by Student's t-test for normally distributed variables and the Mann-Whitney $\mathrm{U}$ test for non-normally distributed variables. Time-to-event variables will be analysed by survival analysis, with the difference between groups assessed with log-rank test.

Post-hoc analyses were be performed by stratifying the patients with intra-axial versus extra-axial tumour, tumour located in supratentorial versus infratentorial region, and craniotomy via frontal versus non-frontal approach.

Statistical analysis will be performed using SPSS V.20.0 and SAS V.9.2 software (SAS Institute). A p value of less than 0.05 is considered statistically significant.

\section{Ethics and dissemination}

The study protocol was approved by the Institutional Review Board of Beijing Tiantan Hospital Affiliated to Capital Medical University (No KY2019-091-02) and registered at ClinicalTrials.gov. The results of the trial will be presented at national and international conferences relevant to subject fields and submitted to international peerreviewed journals.

\section{Summary}

This multicentre, randomised, placebo-controlled, double-blinded trial is designed to investigate the effect of prophylactic use of low-dose dexmedetomidine on the incidence of POD in adult patients admitted to the ICU after intracranial operation for brain tumours.

In the present study, the CAM-ICU is used for the POD diagnosis because all enrolled patients will be admitted to the ICU for postoperative care. In our previous cohort study with 800 neurosurgical patients admitted to the ICU after intracranial operations, $28 \%$ of the patients remained endotracheal intubation. ${ }^{8}$ The CAM-ICU has been validated in mechanically ventilated patients ${ }^{20}$ and non-intubated ICU patients. ${ }^{21}$ The Chinese version of the CAM-ICU has been validated in the ICU setting in mainland China, ${ }^{22}$ and the feasibility has been established in studies reported by our group and others. ${ }^{8} 17$ The CAM-ICU is recommended as a delirium screening tool by the American Society of Critical Care Medicine in adult ICU patients ${ }^{23} 27$ and by the European Society of Anaesthesiology in postoperative patients. ${ }^{1}$ We will conduct the screening of delirium two times per day from postoperative day 1 to day 5 , which is in accordance with the recommendation provided by the European Society of Anaesthesiology evidencebased and consensus-based guideline on POD. ${ }^{1}$ The first POD assessment is performed in the morning on postoperative day 1 , which will be around 24 hours after the operation. This timing of the first assessment is selected to avoid the bias resulting from the diagnosis of emergence delirium, which may be only related to the influence of hypnotics. ${ }^{529}$ Furthermore, investigators in each participating ICU who are in charge of postoperative follow-up are trained by a psychiatrist before the initiation of the study to guarantee the quality of the CAM-ICU evaluation.

We design a low-dose dexmedetomidine infusion $(0.1$ $\mu \mathrm{g} / \mathrm{kg} /$ hour) in the present study to avoid drug-related haemodynamic adverse events such as severe bradycardia demonstrated in our previous study. ${ }^{16}$ The efficacy and safety of this dexmedetomidine dosing regimen have been validated in previous studies in elderly patients after non-cardiac surgery. ${ }^{30}$

The main strength of the present study is the design of a multicentre, randomised, placebo-controlled and double-blinded trial with a relatively large sample size. To the best of our knowledge, this is the first study to evaluate the impact of pharmacological interventions on POD in neurosurgical patients admitted to ICU. The results of the study will provide high-quality evidence. There are also several limitations to our study. First, we will only enrol patients admitted to the ICU, which may represent a population at high risk of POD..$^{3-5}$ Thus, our results may be limited for generalising to the entire population of patients undergoing intracranial surgery. Second, we only evaluate early outcomes up to 28 days after the operation. Third, although we exclude patients with medical records documented preoperative history of mental or cognitive disorders, no systematic cognitive assessment will be performed before the intracranial operation. Forth, the haemodynamic effects of dexmedetomidine such as bradycardia might weaken the efficiency of masking to the treating physicians in the ICU. However, investigators who are responsible for follow-up will not involve in the study agent infusion and patient care. Additionally, the exchange of patient's information is not allowed between investigators in charge of follow-up and ICU physicians taking care of the patients. This will decrease the risk of unmasking to the greatest extent.

\section{Trial status}

At the time of manuscript submission, the study is in the preparation phase for recruitment.

\section{Author affiliations}

${ }^{1}$ Department of Critical Care Medicine, Beijing Tiantan Hospital, Capital Medical University, Beijing, China

${ }^{2}$ China National Clinical Research Center for Neurological Diseases, Beijing Tiantan Hospital, Capital Medical University, Beijing, China

${ }^{3}$ Department of Neurosurgical Critical Care, Xuanwu Hospital, Capital Medical University, Beijing, China

${ }^{4}$ Department of Critical Care Medicine, Tsinghua University Affiliated Beijing Tsinghua Changgung Hospital, Beijing, China

${ }^{5}$ Department of Critical Care Medicine, Peking University Third Hospital, Beijing, China 
Contributors $\mathrm{J}-\mathrm{XZ}$ planned the study. HG performed the statistical design of the study. All authors contributed to the design and development of the trial (XH, K-MC, $L Z, H G, X Q, Y X, P M$ and J-XZ). XH and J-XZ drafted the manuscript. LZ critically revised the manuscript. All authors read and approved the final manuscript.

Funding The study was supported by a grant from the Chinese Stroke Association (CSA2019KY005). The sponsors have no role in the study design and conduct; the data collection, management, analysis and interpretation; or the preparation and approval of the manuscript.

Competing interests The experimental agents (dexmedetomidine hydrochloride and normal saline) are manufactured, packed and provided by the Jiangsu Nhwa Pharmaceutical Co., (Jiangsu, China). Agents provider has no role in the study design and conduct; the data collection, management, analysis and interpretation; or the preparation and approval of the manuscript.

Patient and public involvement Patients and/or the public were not involved in the design, or conduct, or reporting, or dissemination plans of this research.

Patient consent for publication Next of kin consent obtained.

Provenance and peer review Not commissioned; externally peer reviewed.

Open access This is an open access article distributed in accordance with the Creative Commons Attribution Non Commercial (CC BY-NC 4.0) license, which permits others to distribute, remix, adapt, build upon this work non-commercially, and license their derivative works on different terms, provided the original work is properly cited, appropriate credit is given, any changes made indicated, and the use is non-commercial. See: http://creativecommons.org/licenses/by-nc/4.0/.

\section{ORCID iDs}

Hongqiu Gu http://orcid.org/0000-0003-1608-1856

Penglin Ma http://orcid.org/0000-0002-8265-2947

Jian-Xin Zhou http://orcid.org/0000-0002-1559-7554

\section{REFERENCES}

1 Aldecoa C, Bettelli G, Bilotta F, et al. European society of anaesthesiology evidence-based and consensus-based guideline on postoperative delirium. Eur J Anaesthesiol 2017;34:192-214.

2 Mahanna-Gabrielli E, Schenning KJ, Eriksson LI, et al. State of the clinical science of perioperative brain health: report from the American Society of Anesthesiologists brain health Initiative Summit 2018. Br J Anaesth 2019;123:464-78.

3 Whitlock EL, Vannucci A, Avidan MS. Postoperative delirium. Minerva Anestesiol 2011;77:448-56.

4 Inouye SK, Westendorp RGJ, Saczynski JS. Delirium in elderly people. Lancet 2014;383:911-22.

5 Olotu C. Postoperative neurocognitive disorders. Curr Opin Anaesthesiol 2020;33:101-8.

6 Teitelbaum JS, Ayoub O, Skrobik Y. A critical appraisal of sedation, analgesia and delirium in neurocritical care. Can J Neurol Sci 2011;38:815-25

7 Yu A, Teitelbaum J, Scott J, et al. Evaluating pain, sedation, and delirium in the neurologically critically ill-feasibility and reliability of standardized tools: a multi-institutional study. Crit Care Med 2013;41:2002-7.

8 Wang C-M, Huang $\mathrm{H}-\mathrm{W}$, Wang $\mathrm{Y}-\mathrm{M}$, et al. Incidence and risk factors of postoperative delirium in patients admitted to the ICU after elective intracranial surgery: a prospective cohort study. Eur $J$ Anaesthesiol 2020;37:14-24.

9 Chen $\mathrm{H}$, Jiang $\mathrm{H}$, Chen $\mathrm{B}$, et al. The incidence and predictors of postoperative delirium after brain tumor resection in adults: a crosssectional survey. World Neurosurg 2020;140:e129-e139.

10 Budènas A, Tamašauskas Šarūnas, Šliaužys A, et al. Incidence and clinical significance of postoperative delirium after brain tumor surgery. Acta Neurochir 2018;160:2327-37.
11 Flanigan PM, Jahangiri A, Weinstein D, et al. Postoperative delirium in glioblastoma patients: risk factors and prognostic implications. Neurosurgery 2018;83:1161-72.

12 Duan X, Coburn M, Rossaint R, et al. Efficacy of perioperative dexmedetomidine on postoperative delirium: systematic review and meta-analysis with trial sequential analysis of randomised controlled trials. Br J Anaesth 2018;121:384-97.

13 Wu M, Liang Y, Dai Z, et al. Perioperative dexmedetomidine reduces delirium after cardiac surgery: a meta-analysis of randomized controlled trials. J Clin Anesth 2018;50:33-42.

14 Zeng H, Li Z, He J, et al. Dexmedetomidine for the prevention of postoperative delirium in elderly patients undergoing noncardiac surgery: a meta-analysis of randomized controlled trials. PLoS One 2019;14:e0218088.

15 Magoon R, Kumar AK, Malik V, et al. Dexmedetomidine and postoperative delirium: decoding the evidence! J Anaesthesiol Clin Pharmacol 2020;36:140-1.

16 Zhao L-H, Shi Z-H, Chen G-Q, et al. Use of dexmedetomidine for prophylactic analgesia and sedation in patients with delayed extubation after craniotomy: a randomized controlled trial. $J$ Neurosurg Anesthesiol 2017;29:132-9.

17 Su X, Meng Z-T, Wu X-H, et al. Dexmedetomidine for prevention of delirium in elderly patients after non-cardiac surgery: a randomised, double-blind, placebo-controlled trial. Lancet 2016;388:1893-902.

18 Skrobik Y, Duprey MS, Hill NS, et al. Low-Dose nocturnal dexmedetomidine prevents ICU delirium. A randomized, placebocontrolled trial. Am J Respir Crit Care Med 2018;197:1147-56.

19 Chan A-W, Tetzlaff JM, Altman DG, et al. Spirit 2013 statement: defining standard protocol items for clinical trials. Ann Intern Med 2013;158:200-7.

20 Ely EW, Inouye SK, Bernard GR, et al. Delirium in mechanically ventilated patients: validity and reliability of the confusion assessment method for the intensive care unit (CAM-ICU). JAMA 2001;286:2703-10.

21 Ely EW, Margolin R, Francis J, et al. Evaluation of delirium in critically ill patients: validation of the confusion assessment method for the intensive care unit (CAM-ICU). Crit Care Med 2001;29:1370-9.

22 Wang $\mathrm{C}, \mathrm{Wu}$ Y, Yue P, et al. Delirium assessment using confusion assessment method for the intensive care unit in Chinese critically ill patients. J Crit Care 2013;28:223-9.

23 Barr J, Fraser GL, Puntillo K, et al. Clinical practice guidelines for the management of pain, agitation, and delirium in adult patients in the intensive care unit. Crit Care Med 2013;41:263-306.

$24 \mathrm{Wu}$ Y-X, Chen $\mathrm{H}$, Li Q, et al. The prophylactic use of remifentanil for delayed extubation after elective intracranial operations: a prospective, randomized, double-blinded trial. J Neurosurg Anesthesiol 2017;29:281-90.

25 Huang H-W, Yan L-M, Yang Y-L, et al. Bi-frontal pneumocephalus is an independent risk factor for early postoperative agitation in adult patients admitted to intensive care unit after elective craniotomy for brain tumor: a prospective cohort study. PLoS One 2018;13:e0201064.

26 Shan K, Cao W, Yuan Y, et al. Use of the critical-care pain observation tool and the bispectral index for the detection of pain in brain-injured patients undergoing mechanical ventilation: a STROBEcompliant observational study. Medicine 2018;97:e10985.

27 Devlin JW, Skrobik Y, Gélinas C, et al. Clinical practice guidelines for the prevention and management of pain, Agitation/Sedation, delirium, immobility, and sleep disruption in adult patients in the ICU. Crit Care Med 2018;46:e825-73.

28 Ritmala-Castren M, Lakanmaa R-L, Virtanen I, et al. Evaluating adult patients' sleep: an integrative literature review in critical care. Scand $J$ Caring Sci 2014;28:435-48.

29 Munk L, Andersen G, Møller AM. Post-Anaesthetic emergence delirium in adults: incidence, predictors and consequences. Acta Anaesthesiol Scand 2016;60:1059-66.

30 Shen Q-H, Li H-F, Zhou X-Y, et al. Dexmedetomidine in the prevention of postoperative delirium in elderly patients following noncardiac surgery: a systematic review and meta-analysis. Clin Exp Pharmacol Physiol 2020;47:1333-41. 